\title{
UNIVERSITY OF PENNSYLVANIA DATES VII
}

\author{
ELIZABETH K. RALPH, HENRY N. MICHAEL \\ and JOHN GRUNINGER, JR.
}

Department of Physics, University of Pennsylvania, Philadelphia 4, Pa.

\section{INTRODUCTION}

Measurements of samples of known age have been undertaken in this laboratory since its beginning in 1951 when it was found that modern wood samples taken from the outer rings of young trees did not afford a basis of counter calibration consistent with archaeologically dated samples (Ralph, 1955 ). This depletion was explained by Suess (1955). Subsequent measurcments of samples representative of the early Egyptian dynasties, however, revealed that even with the counter calibration corrected for the modern depletion, there was a discrepancy between $\mathrm{C}^{14}$ dates and the early Egyptian chronology (Arnold and Libby, 1951; deVries, Barendsen, and Waterbolk, 1958; deVries and Waterbolk, 1958; Barker and Mackay, 1959, 1961 ; Ralph, 1959; Damon and Long, 1962). Obviously, there was, and still is, a need to find samples of known age for this time range. Dendrochronology may ultimately provide the answer, and in the meantime tree-ring dated samples have afforded a means of assessing the atmospheric $\mathrm{C}^{14}$ inventory during the last $3000 \mathrm{yr}$.

With financial support from the National Science Foundation, which we gratefully acknowledge, we undertook a program of dating sections of Sequoia and bristlecone pine trees both by dendrochronology and by $\mathrm{C}^{14}$. We are indebted to the Laboratory of Tree-Ring Research, University of Arizona, for assistance and collaboration with the tree-ring dating.

The tree-ring dating of the Sequoia sections was readily completed, but that of the bristlecone pines is still in process. $\mathrm{C}^{14}$ dates have helped to accelerate the latter, however, by defining the range of possible tree-ring correlations and have demonstrated also that living bristlecone pine trees extend back 4000 $\mathrm{yr}$. It is hoped that this range may be extended back another 1000 to $2000 \mathrm{yr}$ with "floaters," trees that died years ago but which remain in good condition on the forest floor. It is for these reasons that the $\mathrm{C}^{14}$ results in this list are presented as dates for samples of known age for the Sequoias, that is, as per mil deviations in counting rates. The majority of the bristlecone pines, however, have been treated as samples of unknown age.

All Sequoia samples and bristlecone pines from section P-SW-INY-11A have been corrected for deviations in $\mathrm{C}^{13} / \mathrm{C}^{12}$ ratios. For the few Sequoia samples which were dated before a mass spectrograph was available, the average value for the section has been used for this correction. The standard statistical uncertainty of the average values of the $\mathrm{C}^{13} / \mathrm{C}^{12}$ measurements for each section has been added to that of the $\mathrm{C}^{14}$ to obtain the uncertainty in the $\Delta$ value. The $\delta C^{13}$ values listed represent the deviations (multiplied by 2 ) of the samples measured from the $\delta \mathrm{C}^{13}$ value of our $100-\mathrm{yr}$ old standard oak sample which is also the reference value for the calculation of $\delta \mathrm{C}^{14}$. The $\delta \mathrm{C}^{13}$ relationship between our standard oak sample and NBS limestone standard 


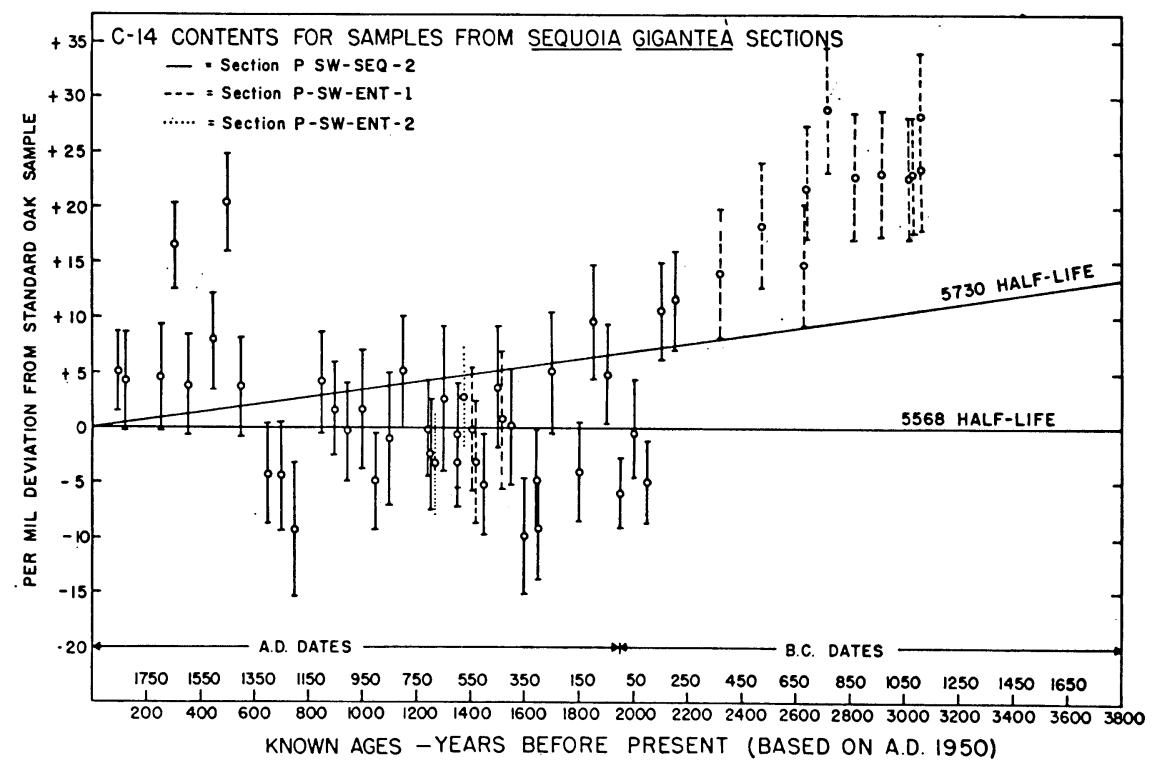

Fig. 1. Per mil deviations from 100-yr-old oak standard of dendrochronologically dated sequoia samples. All have been corrcted for $\mathrm{C}^{13} / \mathrm{C}^{12}$ variations.

\#20 is $-25.7 \pm 1.3$ mils as measured on the University of Pennsylvania mass spectrograph.

It is seen in Tables 1 and 2 that most of the values of $2 \times \delta \mathrm{C}^{13}$ do differ slightly from the $\delta \mathrm{C}^{13}$ value of our 100 -yr old standard oak sample. It must be remembered, however, that these figures are $2 \times \delta \mathrm{C}^{13}$. For comparison with Craig's (1954) data, let us take the average of the three averages $(-7.8 \pm 1.9$, $-8.2 \pm 3.8$, and $-6.3 \pm 1.3)$ and divide by 2 . We obtain $\delta \mathrm{C}^{13}=-3.7 \pm 1.6$ mils as our average deviation for Sequoias from our oak standard. In relation to the NBS \#20 limestone standard this deviation would then be -22 mils which is in excellent agreement with the average value of Craig's determinations. Also, the data exhibit a small irregular scatter as do Craig's. Ours is somewhat larger but our instrument is much less precise. The reason is not clear, however, for the small significant difference in $\delta \mathrm{C}^{13}$ between the Sequoias and our oak standard which is close to the accepted average deviation of woods from limestone ( -25 mils $)$.

The Sequoia results are plotted in Fig. 1. One sees evidence of the oscillations noted by de Vries (1958), Broecker, Olson, and Bird (1959), Willis, Tauber, and Munnich (1960), Suess (1961), and Damon (1963) in the range of A.D. 1700 to 1400 while $\mathrm{C}^{14}$ contents from A.D. 1100 to 100 B.C. cluster consistently just below the 5730 half-life line. A divergence (recorded also by Suess, Damon, and others), which seems to increase with age, is then apparent in the earlier period. The average deviation of the samples representative of the period 650 B.c. to 1150 B.c. represents a difference of approx. $2.3 \%$, or $200 \mathrm{yr}$ in B.P. ages calculated with the 5568 half-life. 
Descriptions of the sections and lists of dates follow. In these lists $\mathrm{C}^{14}$ dates and deviations have been calculated with the 5568 half-life, and B.P. dates are based on A.D. 1950. The uncertainties quoted for the dendrochronologically determined ages represent the age spans of the samples cut out for processing. Since the true dates are close to the median values listed, no allowance for these small spans has been made in the calculations.

\section{Sequoia series (P-SW-SEQ-2)}

Radial sec. of Sequoia gigantea, felled in 1950, from Giant Forest $\left(36^{\circ} 35^{\prime}\right.$ N Lat, $118^{\circ} 48^{\prime}$ W Long), Sequoia Natl. Park, California. Sec. from part of

TABLE 1

\section{P-SW-SEQ-2 Sequoia gigantea}

\begin{tabular}{|c|c|c|c|c|c|}
\hline Lab. No. & $\begin{array}{c}\text { Dendrochronologically } \\
\text { Determined Age } \\
\text { A.D.-B.C. }\end{array}$ & $\begin{array}{l}\text { y } \\
\text { (19.P. } \\
\text { (1950) }\end{array}$ & $\begin{array}{c}\delta 14 \\
\text { (deviation from } \\
\text { Oak St'd mils) }\end{array}$ & $\begin{array}{c}2 \times \delta \mathrm{C}^{13} \\
\text { (deviation from } \\
\text { Oak St'd mils) }\end{array}$ & $\begin{array}{c}\Delta \\
\text { ( } \delta 14 \text { corrected } \\
\text { for } \delta 13 \text { mils) } \\
\text { ones without } \delta \mathrm{C}^{13} \\
\text { are corrected } \\
\text { with average } \\
\text { value of } \delta \mathrm{C}^{13}\end{array}$ \\
\hline P.494 & $200 \pm 5$ в.с. & 2150 & $+17.92 \pm 4.12$ & -6.2 & $+11.6 \pm 4.5$ \\
\hline P.495 & $150 \pm 5$ в.с. & 2100 & $+17.95 \pm 4.02$ & -7.2 & $+10.6 \pm 4.4$ \\
\hline P.496 & $100 \pm 5$ в.с. & 2050 & $+2.97 \pm 3.21$ & & $-4.9 \pm 3.7$ \\
\hline P.497 & $50 \pm 5$ в.с. & 2000 & $+9.05 \pm 4.03$ & -9.0 & $-0.1 \pm 4.4$ \\
\hline P-375 & A.D. $1 \pm 8$ & 1950 & $+2.00 \pm 2.60$ & & $-5.9 \pm 3.2$ \\
\hline P-498 & A.D. $50 \pm 5$ & 1900 & $+12.73 \pm 4.06$ & & $+4.8 \pm 4.5$ \\
\hline P.499 & A.D. $100 \pm 5$ & 1850 & $+15.10 \pm 4.83$ & -5.4 & $+9.6 \pm 5.2$ \\
\hline P.606 & A.D. $150 \pm 5$ & 1800 & $+1.43 \pm 4.13$ & -5.4 & $-4.0 \pm 4.5$ \\
\hline P. $396 \mathrm{~A}$ & A.D. $250 \pm 8$ & 1700 & $+13.90 \pm 5.14$ & -8.8 & $+5.0 \pm 5.5$ \\
\hline P-607 & A.D. $300 \pm 5$ & 1650 & $-2.15 \pm 4.00$ & -7.2 & $-9.4 \pm 4.4$ \\
\hline P-397A & A.D. $302 \pm 10$ & 1648 & $+3.00 \pm 4.06$ & & $-4.8 \pm 4.5$ \\
\hline P-608 & A.D. $350 \pm 5$ & 1600 & $-2.06 \pm 4.93$ & & $-9.9 \pm 5.3$ \\
\hline P-609 & A.D. $400 \pm 5$ & 1550 & $+5.48 \pm 4.97$ & -5.4 & $+0.1 \pm 5.3$ \\
\hline P.610 & A.D. $450 \pm 5$ & 1500 & $+7.74 \pm 5.21$ & -4.0 & $+3.7 \pm 5.5$ \\
\hline P-589B & A.D. $500 \pm 8$ & 1450 & $+3.59 \pm 4.18$ & -8.8 & $-5.2 \pm 4.6$ \\
\hline P. $638 \mathrm{~A}$ & A.D. $600 \pm 8$ & 1350 & $+7.20 \pm 4.40$ & & $-0.7 \pm 4.8$ \\
\hline P.812 & A.D. $600 \pm 7$ & 1350 & $+4.92 \pm 3.73$ & -8.1 & $-3.2 \pm 4.1$ \\
\hline P.643 & A.D. $650 \pm 5$ & 1300 & $+9.84 \pm 6.29$ & -7.2 & $+2.6 \pm 6.6$ \\
\hline P-813 & A.D. $700 \pm 10$ & 1250 & $+5.46 \pm 4.73$ & & $-2.4 \pm 5.1$ \\
\hline P.387 & A.D. $702 \pm 10$ & 1248 & $\begin{array}{r}+7.67 \pm 4.00 \\
\end{array}$ & & $-0.1 \pm 4.4$ \\
\hline P.644 & A.D. $800 \pm 5$ & 1150 & $+12.96 \pm 4.72$ & & $+5.1 \pm 5.0$ \\
\hline P-645 & A.D. $850 \pm 5$ & 1100 & $+6.71 \pm 5.83$ & & $-1.1 \pm 6.1$ \\
\hline P.703 & A.D. $900 \pm 8$ & 1050 & $+2.15 \pm 3.88$ & -7.0 & $-4.9 \pm 4.3$ \\
\hline P. 646 & A.D. $950 \pm 5$ & 1000 & $+12.33 \pm 5.09$ & -10.6 & $+1.6 \pm 5.4$ \\
\hline P.634 & A.D. $1003 \pm 11$ & 947 & $\begin{array}{r}+8.55 \pm 4.19\end{array}$ & -8.8 & $-0.3 \pm 4.6$ \\
\hline P-551 & A.D. $1052 \pm 10$ & 898 & $+9.59 \pm 3.81$ & & $+1.7 \pm 4.3$ \\
\hline P.633 & A.D. $1100 \pm 8$ & 850 & $\begin{array}{r}9.55 \pm 4.22 \\
+\end{array}$ & -5.4 & $+4.1 \pm 4.6$ \\
\hline P.632 & A.D. $1200 \pm 8$ & 750 & $-1.53 \pm 5.74$ & & $-9.3 \pm 6.1$ \\
\hline P-549 & A.D. $1250 \pm 7$ & 700 & $+3.48 \pm 4.5 \mathrm{l}$ & & $-4.4 \pm 4.9$ \\
\hline P.701 & A.D. $1299 \pm 7$ & 651 & $+4.68 \pm 4.11$ & -8.8 & $-4.2 \pm 4.5$ \\
\hline P.700 & A.D. $1400 \pm 8$ & 550 & $+12.80 \pm 4.09$ & -9.0 & $+3.7 \pm 4.5$ \\
\hline P. 647 & A.D. $1450 \pm 10$ & 500 & $+28.38 \pm 4.45$ & -7.8 & $+20.4 \pm 4.5$ \\
\hline P-631 & A.D. $1505 \pm 13$ & 445 & $+16.89 \pm 4.21$ & -8.8 & $+7.9 \pm 4.6$ \\
\hline P. 630 & A.D. $1597 \pm 5$ & 353 & $+12.67 \pm 4.22$ & -8.8 & $+3.8 \pm 4.6$ \\
\hline P. 545 & A.D. $1646 \pm 4$ & 304 & $+24.52 \pm 3.42$ & & $+16.5 \pm 3.9$ \\
\hline P.702 & A.D. $1697 \pm 5$ & 253 & $+15.39 \pm 4.37$ & -10.6 & $+4.6 \pm 4.8$ \\
\hline P-346 & A.D. $1829 \pm 13$ & 121 & $+12.13 \pm 4.10$ & & $+4.2 \pm 4.5$ \\
\hline P.543A & A.D. $1855 \pm 13$ & 95 & $+16.20 \pm 3.09$ & -10.9 & $+5.1 \pm 3.6$ \\
\hline
\end{tabular}


trunk ca. 20 to $25 \mathrm{ft}$ above forest floor while tree was standing. Coll. 1959 and subm. by H. N. Michael, Univ. Mus., Univ. of Pennsylvania. Comment: sec. (P-SW-SEQ-2) was tree ring-dated at Lab. of Tree-Ring Research, Univ. of Arizona, Tucson. Its age range is 212 B.C. to A.D. 1946. Sec. adjacent to this radial sec. was dated by Arizona Radiocarbon Lab. (see Arizona IV, A-255: 1 to 7 ). Measurements of samples from this sec. are listed in Table 1.

\section{Sequoia series (P-SW-ENT-1)}

Radial sec. of Sequoia gigantea from Enterprise Mill area of Balch Park (36 $16^{\circ}$ N Lat, $118^{\circ} 41^{\prime}$ W Long), Mountain Home State Forest, California. Tree probably felled in 1874. Radial sec. came from "butt log" of the tree, a part of the trunk ca. $20 \mathrm{ft}$ above the forest floor while the tree was standing. Butt $\log$ labeled D-22A by Douglass (1919, 1945). Coll. 1960 and subm. by H. N. Michael. Comment: this Sequoia sec. was cross-dated with other Sequoia samples in possession of the Radiocarbon Lab. (samples dated previously by Lab. of Tree-Ring Research, Tucson). The cross-dating was further checked with published data of various sensitive records (Douglass, 1936, 1949). Rings of the sec. cover age span of 1121 B.C. to A.D. 1691. Measurements of these samples listed in Table 2 .

\section{Sequoia series (P-SW-ENT-2)}

Radial sec. of Sequoia gigantea from Enterprise Mill area of Balch Park ( $36^{\circ} 13^{\prime} \mathrm{N}$ Lat, $118^{\circ} 41^{\prime} \mathrm{W}$ Long), Mountain Home State Forest, California.

TABLE 2

P-SW-ENT-1 Sequoia gigantea

\begin{tabular}{|c|c|c|c|c|c|}
\hline Lab. No. & $\begin{array}{c}\text { Dendrochronologically } \\
\text { Determined Age } \\
\text { A.D.-B.c. }\end{array}$ & (1950) & $\begin{array}{l}\quad \delta 14 \\
\text { (deviation from } \\
\text { Oak St'd mils) }\end{array}$ & $\begin{array}{c}2 \times \delta \mathrm{C}^{13} \\
\begin{array}{c}\text { (deviation from } \\
\text { Oak St'd mils) }\end{array}\end{array}$ & $\begin{array}{l}\text { ( } \delta 14 \text { corrected } \\
\text { for } \delta 13 \text { mils) } \\
\text { ones without } \delta \mathrm{C}^{13} \\
\text { are corrected } \\
\text { with average } \\
\text { value of } \delta \mathrm{C}^{13}\end{array}$ \\
\hline P-668 & $1117 \pm 4$ в.с. & 3067 & $+32.36 \pm 3.73$ & -8.7 & $+23.4 \pm 5.3$ \\
\hline P.780 & $1109 \pm 3$ в.с. & 3059 & $+35.66 \pm 4.11$ & -7.1 & $+28.4 \pm 5.7$ \\
\hline P.450 & $1082 \pm 8$ в.с. & 3032 & $+31.47 \pm 3.56$ & & $+23.0 \pm 5.2$ \\
\hline P-667 & $1068 \pm 5$ в.с. & 3018 & $+28.18 \pm 4.09$ & -5.3 & $+22.7 \pm 5.6$ \\
\hline P-665 & $968 \pm 5$ в.с. & 2918 & $+42.81 \pm 4.25$ & -18.9 & $+23.1 \pm 5.7$ \\
\hline P.663 & $868 \pm 5$ в.с. & 2818 & $+28.78 \pm 4.19$ & -5.8 & $+22.8 \pm 5.7$ \\
\hline P-661 & $768 \pm 5$ в.с. & 2718 & $+36.23 \pm 4.18$ & -7.1 & $+28.9 \pm 5.7$ \\
\hline P.449 & $688 \pm 4$ в.с.. & 2638 & $+30.25 \pm 4.09$ & & $+21.8 \pm 5.6$ \\
\hline P.448 & $679 \pm 5$ в.с. & 2629 & $+23.19 \pm 4.09$ & & $+14.8 \pm 5.6$ \\
\hline P.491 & $520 \pm 10$ в.с. & 2470 & $+25.60 \pm 4.09$ & -7.0 & $+18.4 \pm 5.6$ \\
\hline P-656 & $368 \pm 5$ в.с. & 2318 & $+24.16 \pm 4.46$ & -9.9 & $+14.0 \pm 5.9$ \\
\hline P.738 & A.D. $436 \pm 11$ & 1514 & $+4.26 \pm 5.20$ & -3.5 & $+0.8 \pm 6.3$ \\
\hline P-636 & A.D. $544 \pm 22$ & 1406 & $+8.78 \pm 4.06$ & -8.8 & $-0.1 \pm 5.6$ \\
\hline P.815 & A.D. $532 \pm 20$ & 1418 & $+4.11 \pm 4.08$ & -7.2 & $-3.1 \pm 5.6$ \\
\hline \multicolumn{6}{|c|}{ P.SW-ENT-2 Sequoia gigantea $=-8.2 \pm 3.8$} \\
\hline P.818 & A.D. 577 & 1373 & $+8.25 \pm 4.27$ & -5.4 & $+2.8 \pm 4.5$ \\
\hline P-821 & A.D. 677 & 1273 & $+10.50 \pm 4.33$ & -7 & $-3.2 \pm 4.6$ \\
\hline
\end{tabular}


Tree probably felled in 1874. Sec. from high stump (ca. $6 \mathrm{ft}$ above forest floor) of a tree labeled D-43 by Douglass (1936). Coll. 1960 and subm. by H. N. Michael. Comment: this Sequoia sec. cross-dated with other Sequoia samples in possession of the Radiocarbon Lab. (samples dated previously by Lab. of Tree-Ring Research). Cross-dating further checked with published data of various sensitive records (Douglass, 1928, 1949). Sec. has age span from 1119 в.C. to A.D. 1699. Measurements of these samples listed in Table 2.

\section{Bristlecone Pine series (P-SW-INY-1)}

Horizontally cut cross-sec. from stump of Pinus aristata from White Mountains, California-Nevada. Tree felled in 1956 and labeled WHTw4779 by Schulman $(1956,1958)$. It grew at elevation of $10,500 \mathrm{ft}\left(37^{\circ} 25^{\prime} 30^{\prime \prime} \mathrm{N}\right.$ Lat, $118^{\circ} 10^{\prime} 20^{\prime \prime} \mathrm{W}$ Long (, approx. $4000 \mathrm{ft} \mathrm{W}$ of Goat Springs. Coll. 1960 and subm. by H. N. Michael. Comment: specimen not fully analyzed or cross-dated. However, ring count and $\mathrm{C}^{14}$ determinations indicate age span slightly in excess of $4000 \mathrm{yr}$.

The following samples from this section were processed:

\begin{tabular}{|c|c|c|}
\hline Lab. No. & $\begin{array}{l}\mathrm{C}^{14} \text { Date } \\
\text { (A.D.-B.C.) }\end{array}$ & $\begin{array}{c}\mathrm{C}^{14} \text { Date } \\
\text { (B.P.) }\end{array}$ \\
\hline P.407 & $2092 \pm 69$ в.с. & $4042 \pm 69$ \\
\hline P.600 & $2056 \pm 52$ в.с. & $4006 \pm 52$ \\
\hline P. 434 & $1988 \pm 51$ в.с. & $3938 \pm 51$ \\
\hline P.435 & $1817 \pm 56$ в.с. & $3767 \pm 56$ \\
\hline P.413 & $1797 \pm 67$ в.с. & $3747 \pm 67$ \\
\hline P.411 & $1722 \pm 66$ в.с. & $3672 \pm 66$ \\
\hline P.602 & $1156 \pm 42$ в.с. & $3106 \pm 42$ \\
\hline P.493 & $1052 \pm 57$ в.с. & $3002 \pm 57$ \\
\hline P.451 & $500 \pm 47$ в.с. & $2450 \pm 47$ \\
\hline P. 430 & $379 \pm 46$ в.с. & $2329 \pm 46$ \\
\hline P. 436 & $327 \pm 42$ в.с. & $2277 \pm 42$ \\
\hline P. 492 & $498 \pm 47$ в.с. & $2448 \pm 47$ \\
\hline P. 433 & A.D. $1323 \pm 56$ & $627 \pm 56$ \\
\hline P. 453 & A.D. $1349 \pm 48$ & $601 \pm 48$ \\
\hline P-431 & A.D. $1294 \pm 40$ & $656 \pm 40$ \\
\hline-416 & $781 \pm 47$ & $169 \pm$ \\
\hline
\end{tabular}

\section{Bristlecone Pines series (P-SW-INY-2)}

Horizontally cut cross-sec. from stump of Pinus aristata from White Mountains, California-Nevada. Tree felled in 1956 and given the Lab. of TreeRing Research acquisition number WHT $_{\mathrm{B}_{2}} 5547$, according to C. W. Ferguson of that lab. Tree grew in "Methuselah Walk" area $\left(37^{\circ} 23^{\prime} 40^{\prime \prime} \mathrm{N}\right.$ Lat, $118^{\circ} 09^{\prime}$ $30^{\prime \prime}$ W Long), ca. $2.2 \mathrm{mi}$ by trail from Schulman Grove. Coll. 1960 and subm. 


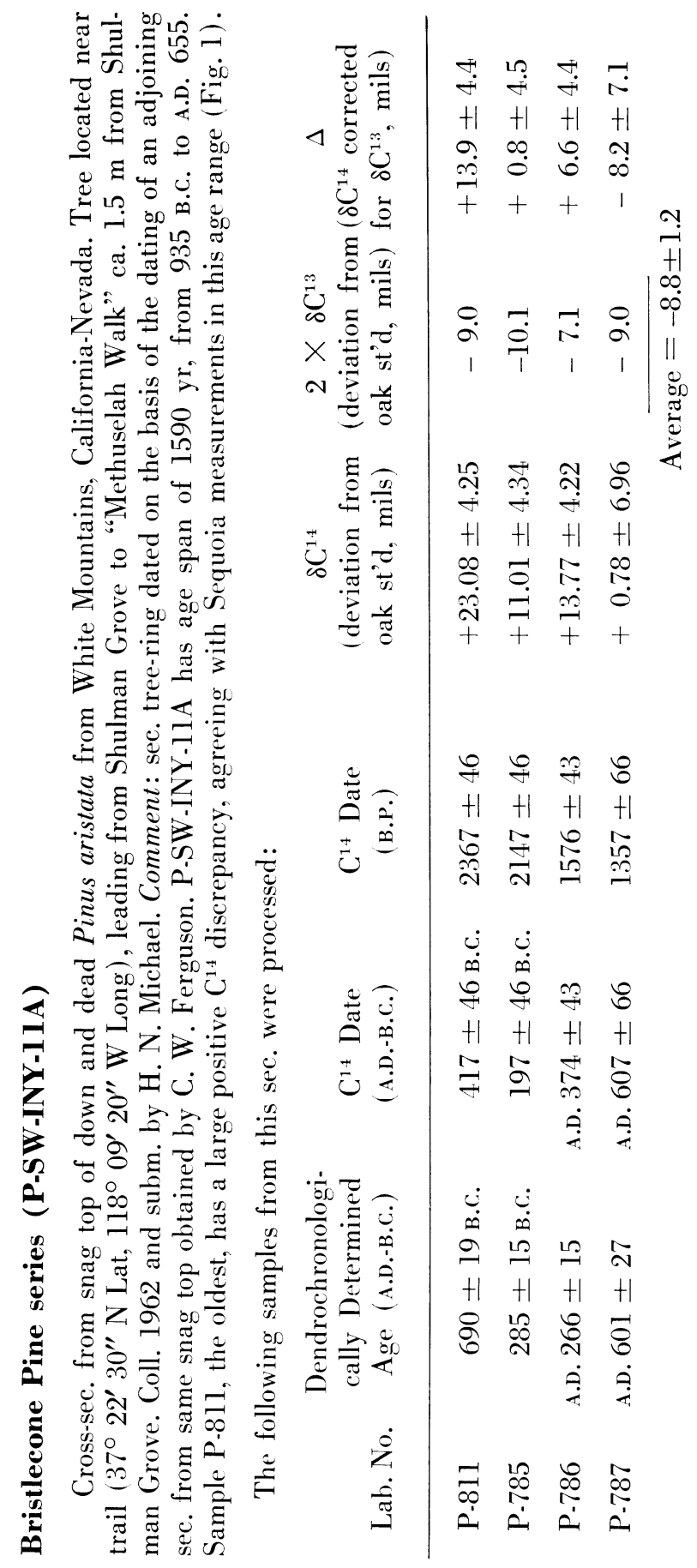


by H. N. Michael. Comment: specimen cross-dated only in its A.D. range.

The following samples from this sec. were processed:

$\begin{array}{ccc} & \begin{array}{c}\text { C'14 Date } \\ \text { Lab. No. }\end{array} & \begin{array}{c}\mathrm{C}^{14} \text { Date } \\ \text { (A.D.-B.c.) }\end{array} \\ \text { P.415 } & 1287 \pm 55 \text { в.C. } & 3237 \pm 55 \\ \text { P.429 } & 882 \pm 44 \text { B.C. } & 2832 \pm 44 \\ \text { P-427 } & 397 \pm 48 \text { в.C. } & 2347 \pm 48 \\ \text { P-428* } & \text { A.D. } 356 \pm 40 & 1594 \pm 40\end{array}$

* The dendrochronologically determined date for this sample is A.D. $345 \pm 20$.

\section{White Mountains, California "floater" series, Bristlecone Pines}

Wood, Pinus aristata, from immediate vicinity of Schulman Grove $\left(37^{\circ}\right.$ $23^{\prime} 15^{\prime \prime}$ N Lat, $118^{\circ} 10^{\prime} 30^{\prime \prime}$ W Long), White Mountains, California. Comment: the three samples described below are "floaters," i.e., weathered pieces of wood picked up from the forest floor which were thought to be chronological predecessors of some of the present trees and which might prove useful in cross-dating with other samples.

P-603. Sec. No. P-SW-INY-13b

$1952 \pm 36$

2 B.C.

Wood, Pinus aristata, from White Mountains $\left(37^{\circ} 23^{\prime} 20^{\prime \prime} \mathrm{N}\right.$ Lat, $118^{\circ}$ $10^{\prime} 40^{\prime \prime} \mathrm{W}$ Long), California. Coll. 1962 and subm. by H. N. Michael.

\section{P-604. Sec. No. P-SW-INY-10 (TRL 62-81b) $2602 \pm 35$} $10^{\prime} 40^{\prime \prime}$ W Long), California Coll. 1962 by C. W. Ferguson, Univ. subm. by H. N. Michael.

\section{P-605. Sec. No. P-SW-INY-9 (TRL 62-80b) $\quad 3225 \pm 47$}

Wood, Pinus aristata, from White Mountains $\left(37^{\circ} 23^{\prime} 05^{\prime \prime} \mathrm{N}\right.$ Lat, $118^{\circ}$ $10^{\prime} 40^{\prime \prime}$ W Long), California. Coll. 1962 by C. W. Ferguson; subm. by H. N. Michael.

Date list:

$$
\text { REFERENCES }
$$

Arizona IV Damon, Long, and Sigalove, 1963

Arnold, J. R., and Libby, W. F., 1951, Radiocarbon dates: Science, v. 113, p. 111.

Barker, H., and Mackey, C. J., 1959, British Museum natural radiocarbon measurements I. Am. Jour. Sci. Radioc. Supp., v. 1, p. 83-85. v. 3, p. $39-45$, cker, W. S., Olson, E.A., and Bird, J., 1959, Radiocarbon measurements on samples of known age: Nature, v. 183, p. 1582-1584.

Craig, H., 1954, Carbon-13 variations in Sequoia rings and the atmosphere: Science, v. 119, p. 141-143.

Damon, P. E., and Long, A., 1962, Arizona radiocarbon dates III: Radiocarbon, v. 4, p. 204-238.

Damon, P. E., 1963, Carbon-14, carbon dioxide and climate: Paper delivered at IUGG 13th Assembly, Berkeley, California.

Damon, P. E., Long, A., and Sigalove, J. J., 1963, Arizona radiocarbon dates IV: Radiocarbon, v. 5 , p. $285-286$. 
Douglass, A. E., 1919, Climatic cycles and tree growth: A study of the annual rings of trees in relation to climatic and solar activity: Carnegie Inst. of Washington pub. 289, v. 1 , p. $44-53$.

1928, Climatic cycles and tree growth, v. II: A study of the annual rings of trees in relation to climate and solar activity: Carnegie Inst. of Washington pub. 289, v. 2, p. 5-56 and p. 139-158.

Douglass, A. E., 1936, Climatic cycles and tree growth, v. III: A study of cycles: Carnegie Inst. of Washington pub. 289, v. 3, p. 3 and p. 99-105. 1945, Survey of sequoia studies: Tree Ring Bull., v. 11, p. 26-32.

1949, A superior sequoia ring record: Tree-Ring Bull., v. 16, p. 2-6.

Ralph, E. K., 1955, Univ. of Pennsylvania radiocarbon dates I; Science, v. 121, p. 149. 1959, Univ. of Pennsylvania radiocarbon dates III: Am. Jour. Sci. Radioc. Supp., v. 1, p. 47-48.

Schulman, E., 1954, Dendroclimatic changes in semiarid regions: Tree-Ring Bull., v. 20, p. 26-30.

1956, Dendroclimatic changes in semiarid America: Tucson, Univ. of Arizona Press, 142 p.

p. 355-372. Bristlecone pine, oldest known living thing: Natl. Geog. Mag., v. 113, p. $355-372$.

Suess, H. E., 1955, Radiocarbon concentration in modern wood: Science, v. 122, p. 415-417. 1961, Secular changes in the concentration of atmospheric radiocarbon: in Problems related to interplanetary matter, proc. of an informal conference, Highland Park, Illinois; Natl. Acad. of Sci., pub. 845, p. 90-95.

Vries, H. de, 1958, Variation in concentration of radiocarbon with time and location on earth: Proc. Kon. Ned. Akad. van Wetenschappen, ser. B, v. 61, p. 1-9.

Vries, H. de, and Waterbolk, H. T., 1958, Groningen radiocarbon dates III: Science, v. 128, p. 1550-1556.

Vries, H. de Barendsen, G. W., and Waterbolk, H. T., 1958, Groningen radiocarbon dates II: Science, v. 127, p. 129-137.

Willis, E. H., Tauber, H., and Munnich, K. O., 1960, Variations in the atmospheric radiocarbon concentration over the past 1300 years: Am. Jour. Sci. Radioc. Supp., v. 2, p. 1-4. 